\title{
The dilemma of complicated shunt valves: How to identify patients with posthemorrhagic hydrocephalus after aneurysmatic subarachnoid hemorrhage who will benefit from a simple valve?
}

\author{
Christian von der Brelie, Ullrich Meier, Alexander Gräwe, Johannes Lemcke \\ Department of Neurosurgery, Neurovascular Centre, Unfallkrankenhaus Berlin, Berlin, Germany
}

\begin{abstract}
Background: Sophisticated shunt valves provide the possibility of pressure adjustment and antisiphon control but have a higher probability of valve dysfunction especially in a posthemorrhagic setting. The aim of the present study is to analyze the clinical outcome of patients with shunt dependent posthemorrhagic hydrocephalus after aneurysmatic subarachnoid hemorrhage (SAH) in order to identify patients who would benefit from a simple differential pressure valve. Methods: From 2000 to 2013, 547 patients with aneurysmatic SAH were treated at our institution, 114 underwent ventricular shunt placement (21.1\%). 47 patients with available pre- and post-operative computed tomography scans, and an available follow-up of minimum 6 months were included. In order to measure the survival time which a nonprogrammable differential pressure valve would have had in an individual patient we defined the initial equalized shunt survival time (IESS). IESS is the time until surgical revisions of fixed differential pressure or flow-regulated valves for the treatment of over- or under-drainage as well as re-programming of adjustable valves due to over- or under-drainage. Results: Twenty patients were treated with fixed differential pressure valves, 15 patients were treated with flow-regulated valves, and 12 underwent ventriculoperitoneal (VP) shunt placement with differential pressure valves assisted by a gravitational unit. Patients who reacted with remarkable changes of the ventricular width after the insertion of external ventricular drainage (EVD), before shunt placement, showed a significantly longer IESS. Conclusions: Decline of the ventricular width after EVD placement was a predictor for successful VP shunt therapy in the later course of disease. Possibly, this could allow identifying patients who benefit from a simple differential pressure valve or a flow-regulated valve, and thus could possibly avoid valve-associated complications of a programmable valve in the later course of disease.
\end{abstract}

Key words: Gravitational unit, outcome, posthemorrhagic hydrocephalus, subarachnoid hemorrhage, valve settings

\section{Introduction}

Posthemorrhagic hydrocephalus ( $\mathrm{pHC}$ ) is a major complication in the clinical course of aneurysmal subarachnoid hemorrhage (SAH) ${ }^{[1,2]}$ A wide range of incidence of $\mathrm{pHC}$ in SAH patients ranging from $6 \%$

\section{Address for correspondence:}

Dr. Johannes Lemcke, Department of Neurosurgery

Unfallkrankenhaus Berlin, Warener Str. 7, 12683 Berlin, Germany.

E-mail: johannes.lemcke@ukb.de

\begin{tabular}{|l|l|}
\hline \multicolumn{2}{|c|}{ Access this article online } \\
\hline Quick Response Code: & Website: \\
\hline & www.ruralneuropractice.com \\
\cline { 2 - 3 } & \\
\hline
\end{tabular}

to $60 \%$ is reported..$^{[2-4]}$ Onset of drainage dependent hydrocephalus may be oftentimes acute and sometimes later in the intermediate course after aneurysm rupture. ${ }^{[5]}$ A variety of independent risk factors for drainage-dependent hydrocephalus after aneurysmal SAH could be identified. These include Hunt and Hess (Hand $\mathrm{H})$ grade, Fishergrade, intraventricularhemorrhage, and angiographic vasospasm. ${ }^{[1]}$ Besides aneurysmal $\mathrm{SAH}, \mathrm{pHC}$ occurs after different posthemorrhagic conditions such as nonaneurysmal SAH, all different

This is an open access article distributed under the terms of the Creative Commons Attribution-NonCommercial-ShareAlike 3.0 License, which allows others to remix, tweak, and build upon the work non-commercially, as long as the author is credited and the new creations are licensed under the identical terms.

For reprints contact: reprints@medknow.com

How to cite this article: Brelie Cv, Meier U, Gräwe A, Lemcke J. The dilemma of complicated shunt valves: How to identify patients with posthemorrhagic hydrocephalus after aneurysmatic subarachnoid hemorrhage who will benefit from a simple valve?. J Neurosci Rural Pract 2016;7:48-54. 
forms of intradural hemorrhage after traumatic brain injury (traumatic subdural hematoma, traumatic SAH, intracerebral hemorrhage, intraventricular hemorrhage), or intracerebral/intraventricular hemorrhage in new borns, each representing its own pathological disease complex which might potentially result in different pathophysiological aspects regarding $\mathrm{pHC}^{[1,6]}$ Pathophysiological mechanisms include changes in the constitution of cerebrospinal fluid (CSF), alterations in CSF circulation, adhesions in the subarachnoid space as well as in the ventricles and the cisterns which result in altered CSF flow dynamics and pulsatility, and reduced CSF absorption at the capillary bed of the cortex and at the arachnoid granulations. ${ }^{[7-10]}$

Placement of ventriculoperitoneal (VP) shunt adequately treats $\mathrm{pHC}$ and reduces morbidity and lethality. Still, there is no widely accepted standard for the optimum interval between the aneurysm rupture and the time of shunt placement. Furthermore, the choice of valve type, the valve opening pressure to establish adequate drainage and uneventful postoperative course in patients with pHC due to aneurysmal SAH, is still difficult.

On the one hand, inadequate opening pressure of nonprogrammable valves may lead to revision surgery; on the other, programmable valves may lead to valve associated complications, for example, mechanical dysfunction. ${ }^{[11-13]}$

Therefore, we analyzed the initial equalized shunt survival (IESS), defined as the period of time in which a VP shunt with any valve type remains in its initial configuration without re-programming or revision surgery. The aim of this retrospective study is to identify predictors of long IESS. The goal would be to enable the neurosurgeon to identify patients with $\mathrm{pHC}$, after aneurysmal SAH who benefit from simple non valves and do not need a valve.

\section{Methods}

\section{Study design}

The hospital's electronic surgical database was retrospectively screened from 2000 to 2013. Patients with acute aneurysmal SAH, who underwent VP shunting in the course for $\mathrm{pHC}$ were identified. The study has been approved by the Institutional Review Board.

\section{Inclusion/exclusion criteria}

Only patients with aneurysmal SAH were included. Patients with perimesencephalic nonaneurysmal SAH and traumatic SAH were explicitly excluded.
Out of 547 patients with aneurysmal SAH, 114 (21.1\%) developed pHC and thus were eligible for this study. Since only patients with a clinical follow-up of at least 6 months and electronic availability of primary and pre- and post-operative computed tomography (CT) scan were included. Overall, 47 patients were included in our analysis.

\section{Data collection}

Besides the baseline data, we collected the following data from the database: The site of the aneurysm was analyzed, the clinical presentation of the patients was evaluated using $\mathrm{H}$ and $\mathrm{H}$ grading. We analyzed whether the ruptured aneurysm was surgically or endovascularly treated. It was noted if and when an external ventricular drain (EVD) was inserted. Further neurosurgical procedures such as decompressive craniectomy, change of EVD, or secondary microsurgical clipping following endovascular treatment were analyzed. It was noted if a temporary lumbar drainage was inserted.

Further, the delay until the definitive VP shunt was analyzed. The time span between the removal of the EVD and the insertion of the VP shunt was checked. The preoperative CSF constitution was analyzed, protein and lactate levels as well as leukocytes/ $\mu$ l CSF were noted. The type of the shunt valve was analyzed as well as if a gravitational unit was used. Shunt valves were then functionally categorized into three groups: (1) Pressure regulated valves (including valveless, nonadjustable pressure valves and diff erential pressure valves), (2) flow-regulated valves, and (3) VP shunts with any valve plus a gravitational unit. The duration of the VP shunt procedure was analyzed. Evans index (EI) as well as the width of the third ventricle were analyzed on the initial CT scan as well as after the insertion of an EVD. ${ }^{[14]}$ The same parameters were analyzed on the last CT scan before VP shunting and at the time point of clinical benefit or clinical well-being, respectively.

Postoperative clinical course was analyzed; surgical interventions due to any VP shunt complications were noted.

The period of time until surgical revisions for treatment of over- or under-drainage as well as re-programming of adjustable valves were necessary was defined as IESS. The clinical outcome after SAH was measured using the Glasgow Outcome Scale score.

Management of patients and presurgical choice of valve In our hospital, all patients with SAH are managed by the Department of Neurosurgery. All patients were admitted via the emergency unit and immediately 
received a native $\mathrm{CT}$ scan and a CT angiography. When admitted before $10 \mathrm{pm}$, patients received conventional cerebral angiogram on the same day, and after $10 \mathrm{pm}$, angiogram was performed the next morning. Patients with suspected elevated intracranial pressure (ICP) due to intracerebral hemorrhage or hydrocephalus were treated immediately. In patients with radiological signs of CSF retention, an EVD was inserted. Once the potentially ruptured aneurysm was detected, an interdisciplinary decision was made to determine the treatment modality. After neurosurgical or interventional treatment, the patients were transferred to the Intensive Care Unit.

CSF drainage was adjusted in a way that $100-150 \mathrm{ml} / 24 \mathrm{~h}$ amount of CSF is drained. In some cases, CSF drainage was also increased in case of elevated ICP, if after follow-up computerized axial tomography (CAT) scan still showed hydrocephalic configuration. Follow-up CAT scan was acquired in the first $24 \mathrm{~h}$ after aneurysm rupture. Depending on the blood load in the CSF and the ICP, weaning of the patients from the EVD was tried by increasing stepwise the opening pressure of the EVD system. In cases of unsuccessful weaning from EVD, a VP shunt system was placed. Fixed differential pressure valve systems were implanted in immobile patients in order to lower the complexity of the implant and thus avoid malfunction of unneeded valve components. Programmable, auto-regulative, or gravitational valves were implanted in mobile patients with anticipated need for posture dependent valve performance. By trend, flow-regulated valves were implanted in cases with rather high drainage volumes and without pathological ICP values at any time and inhomogeneous distribution of the drainage amount over $24 \mathrm{~h}$. Follow-up CAT scan was acquired within the first $24 \mathrm{~h}$ after VP shunt placement.

\section{Statistical analysis}

The Mann-Whitney U-test, Kruskal-Wallis test, Student's two-tailed $t$-test, one-way ANOVA, and Fisher exact test were used to determine statistically significant differences between the patients and different patient groups. Kaplan-Meier survival curves were determined. Log rank test was applied to analyze differences for shunt survival.

Statistical significance was defined as a $P \leq 0.05$. All data were analyzed using GraphPad Software, Inc., La Jolla, CA, USA.

\section{Results}

Patient and subarachnoid hemorrhage characteristics Overall, 47 patients, with acute hemorrhage SAH who received VP shunting in the course, were included in the study. All of these patients had a follow-up of minimum 3 months after SAH. Median age was 51.7 years; 24 patients were male. Eighteen patients $(38.3 \%)$ were clinically presented with $\mathrm{H}$ and $\mathrm{H}$ grade II (21.3\% grade III, $19 \%$ grade IV, $21.3 \%$ grade V) [Table 1]. Twenty-three (49\%) of all ruptured aneurysms were located in the anterior communicating artery complex (19\% middle cerebral artery, $13 \%$ internal carotid/posterior communicating artery, $15 \%$ posterior circulation, and $2 \%$ pericallosal artery). Treatment of the ruptured aneurysm was performed by endovascular coil embolization in $72 \%$, $25 \%$ of all patients underwent surgical clipping. In one patient (85 years, clinical presentation with $\mathrm{H}$ and $\mathrm{H}$ grade II), harboring a large basilar tip aneurysm neither endovascular nor surgical treatment was performed. Initial CT scan revealed Fisher III hemorrhage in 91\% of all patients. Intraventricular blood was seen in $62 \%$ of all patients.

EVD was placed in $94 \%$ of all patients for either the treatment of $\mathrm{pHC}(86 \%$ - EI $>0.3)$ or monitoring purpose $(14 \%)$ as an emergency procedure. In six cases, an infection of the EVD was noted (14\%). In

\begin{tabular}{lc}
$\begin{array}{l}\text { Table 1: Baseline data of } \mathbf{4 7} \text { patients included in the } \\
\text { study }\end{array}$ \\
\hline Patient characteristics & Mean/count \\
\hline Age & (30-85) years \\
Male & 24 \\
Female & 23 \\
SAH characteristics & Percentage \\
MCA & 19.3 \\
AcomA & 49.9 \\
ICA/AcomA & 13.8 \\
Posterior circulation & 14.9 \\
Pericallosal & 2.1 \\
H and H II & 38.3 \\
H and H III & 21.3 \\
H and H IV & 19.1 \\
H and H V & 21.3 \\
& 100 \\
Fisher 1 & 0 \\
Fisher 2 & 2.4 \\
Fisher 3 & 91 \\
Fisher 4 & 6.6 \\
EVD & 94 \\
No EVD & 6 \\
\hline Han H H & 6 \\
\hline
\end{tabular}

$\mathrm{H}$ and $\mathrm{H}$ : Hunt and Hess, EVD: External ventricular drain, MCA: Middle cerebral artery, ICA: Internal carotid 
22 patients (46\%), further neurosurgical procedures were carried out (17 changes of EVD, 7 decompressive hemicraniectomies (DHC); two patients underwent change of EVD as well as DHC) [Table 2].

Ventriculoperitoneal shunt treatment and valve type selection

VP shunt insertion took place in all patients included in this study. Median interval between SAH and shunt placement was 29 days. Mean length of surgical procedure was $55 \mathrm{~min}$. Laboratory investigations of CSF before shunt placement revealed a mean lactate level $3.0 \mathrm{mmol} / \mathrm{l}$, a mean CSF leukocyte count of 41.4 leukocyte/ $\mu \mathrm{l} \mathrm{CSF}$, and the mean protein levels of $449.5 \mathrm{mg} / \mathrm{l}$. Nine patients underwent secondary neurosurgical admission for the placement of shunt after they had been discharged post, primary treatment. Median interval between SAH and shunt placement was 92 days in this subgroup.

Different kinds of shunt valves were used. They were categorized in different subgroups according to the functional principle. Twenty patients were treated with differential pressure valves of all kinds, 15 patients were treated with flow-regulated valves, and 12 underwent VP shunt placement with differential pressure valves assisted by a gravitational unit. The groups did not differ from each other in most of the analyzed parameters [Figure 1]. Nonetheless shunt placement with differential pressure valves took place significantly after a shorter interval after SAH $(P=0.001)$.

The percentage of patients of shunt failures in the different valve groups was not statistically different. Kaplan-Meier curves analyzing shunt failure for shunt survival in the different valve groups did also not show any difference $(P=0.06$; log rank test). Since the flow-regulated valve group showed the smallest shunt failure rate, the group was tested separately against all other patients in a pooled group. Shunt survival was not statistically different in this testing either $(P=0.07$, $\log$ rank test).
Evans Index development after external ventricular drain predicts ventriculoperitoneal shunt initial equalized shunt survival

$\mathrm{CT}$ imaging was performed initially to obtain the diagnosis of SAH; simultaneously width of cerebral ventricles were analyzed for hydrocephalic configuration. Indication for EVD placement due to $\mathrm{pHC}$ and/or invasive monitoring was made in $94 \%$ of all patients. Analyzing EI before and after EVD placement result in a wide range of changes in ventricular width $(\Delta \mathrm{EI}$, median 0.03 , range $-0.02-0.12$ ). Comparing the 30 patients with change of EI more than 0.03 after EVD insertion against the 14 patients with change of EI below 0.03, IESS was significantly longer in patients with change of EI larger than 0.03 [Figure 2a; $P=0.001$ ].

The patients with a $\Delta \mathrm{EI}$ after EVD $<0.03$ underwent VP shunt insertion with different valves. However, numbers of patients in the different valve groups were too small to analyze differences in shunt failure.

$\Delta$ EI was also analyzed after VP shunt placement. Median change of EI was 0.048 (range 0.01-0.11). Comparing the 25 patients with change of $\mathrm{EI}<0.04$ with the 22 patients with change of EI $>0.04$, shunt survival was significantly

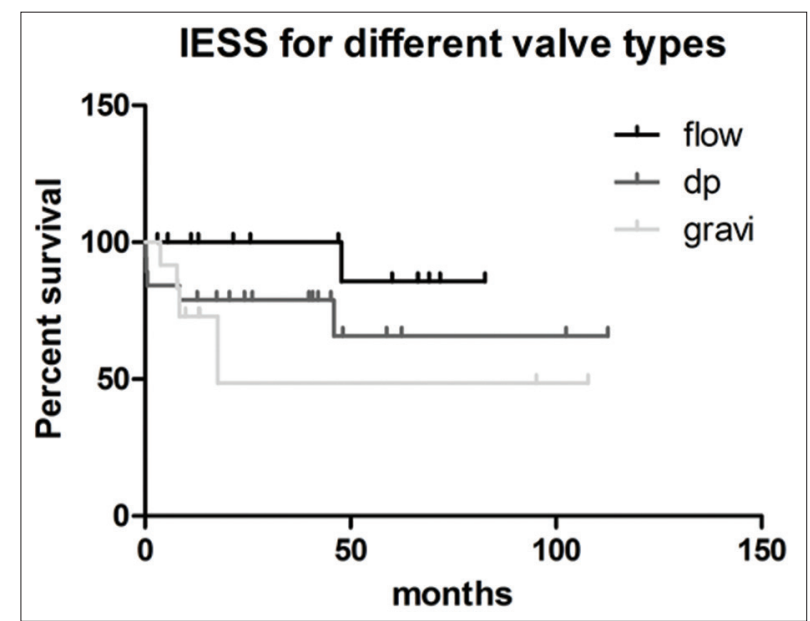

Figure 1: Dependency of the initial equalized shunt survival on the valve type

Table 2: SAH and outcome parameters according to the implanted valve type

\begin{tabular}{|c|c|c|c|c|c|c|c|c|c|}
\hline $\begin{array}{l}\text { Valve functional } \\
\text { principle }\end{array}$ & $\begin{array}{c}\text { Mean } \mathrm{H} \\
\text { and } \mathrm{H}\end{array}$ & Coil/clip & $\begin{array}{c}\text { Interval } \\
\text { between SAH } \\
\text { and VP shunt } \\
\text { insertion (days) }\end{array}$ & $\begin{array}{c}\text { Mean } \\
\text { change in } \\
\text { El after EVD } \\
\text { placement }\end{array}$ & $\begin{array}{c}\text { Mean } \\
\text { change in EI } \\
\text { after shunt } \\
\text { placement }\end{array}$ & $\begin{array}{c}\text { Shunt } \\
\text { failure - } \\
\text { yes/no }\end{array}$ & $\begin{array}{c}\text { Shunt } \\
\text { infection? }\end{array}$ & $\begin{array}{l}\text { Outcome } \\
\text { (mean } \\
\text { GOS) }\end{array}$ & $\begin{array}{c}\text { Median } \\
\text { follow-up } \\
\text { duration }\end{array}$ \\
\hline Differential pressure valve & 3.5 & $\begin{array}{c}11 / 8 \\
\text { (1-not treate }\end{array}$ & 24 & 0.03 & 0.04 & $5 / 15$ & $3 / 20$ & 3.5 & 39.8 \\
\hline Flow-regulated valve & 3.1 & $11 / 3$ & 34 & 0.04 & 0.06 & $2 / 13$ & $3 / 15$ & 3.5 & 47.0 \\
\hline Gravitational valve & 2.8 & $10 / 2$ & 36.5 & 0.03 & 0.04 & $4 / 8$ & $0 / 12$ & 3.6 & 12.9 \\
\hline Statistical significance & $P=0.72^{*}$ & - & $P=0.001^{* *}$ & $P=0.36^{*}$ & $P=0.49^{*}$ & $P=0.46^{*}$ & - & $P=0.94^{*}$ & $P=0.94^{*}$ \\
\hline
\end{tabular}

"not significant, ** significant. Valve type versus IESS. IESS: Initial equalized shunt survival, GOS: Glasgow Outcome Scale, SAH: Subarachnoid hemorrhage,

EVD: External ventricular drain, VP: Ventriculoperitoneal, El: Evans index, $\mathrm{H}$ and $\mathrm{H}$ : Hunt and Hess 


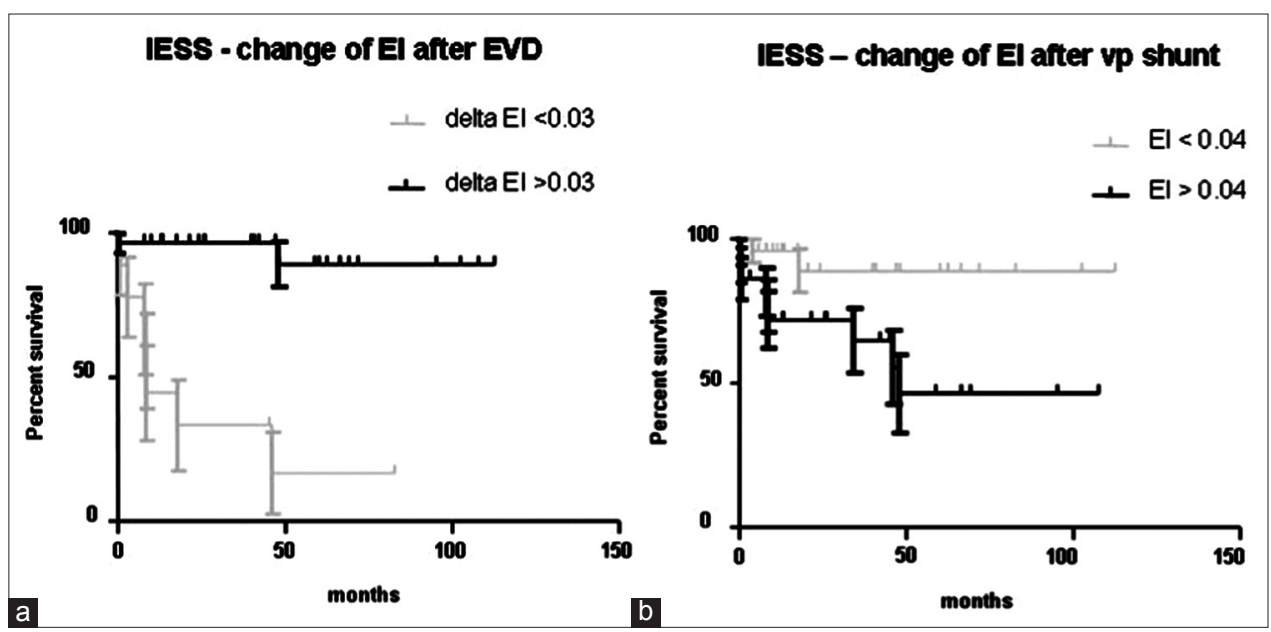

Figure 2: (a) Dependency of the initial equalized shunt survival from initial changes of the Evans index after external ventricular drain placement. (b) Dependency of the initial equalized shunt survival from changes of the Evans index after ventriculoperitoneal shunt placement

better in patients with smaller change of EI [Figure $2 b$, $P=0.02]$. The distribution of valves in the different groups was not statistically different $(P=0.42)$.

\section{Discussion}

Even though programmable valves with additional gravitational functions have been proven to be superior in the therapy of other types of hydrocephalus, the authors doubt that every patient with $\mathrm{pHC}$ after aneurysmatic SAH needs a complex shunt valve. ${ }^{[15,16]}$

To speak from the authors' own experience, complex valves in SAH patients tend to develop malfunctions even if the patient would never have needed any of the complex features. Based on this experience, the authors tried to identify predictors which would allow identifying patients who are perfectly benefited from a simple nonprogrammable differential pressure valve.

Bearing this in mind, we analyzed our patients with $\mathrm{pHC}$ after aneurysm rupture. In order to reflect the necessity of the "first successful shot on the target," we analyzed the IESS. This is a newly defined endpoint for the analysis of the clinical course after shunt surgery. The advantage of this definition is the comparability of programmable and nonprogrammable valve types. The disadvantage is that it equalizes the events of different impacts in terms of invasiveness. However, since these events might have comparable impacts on patients' lives, this simplification seems to be justifiable to the authors.

\section{Evans index versus initial equalized shunt survival} Analyzing the dynamics of the ventricular system in terms of changes of the EI, we observed effects on the IESS which appeared as paradox at first sight:
- Patients who reacted with remarkable changes of the ventricular width $(\Delta \mathrm{EI}>0.03)$ during the EVD showed a longer IESS

- Patients who reacted with no changes of the ventricular width $(\Delta \mathrm{EI}<0.04)$ after VP shunt placement showed a longer IESS.

Finding two is analog to the results of an analysis of patients with idiopathic normal pressure hydrocephalus (iNPH). ${ }^{[17]}$ Unless the pathophysiology of neither iNPH nor pHC is unriddled, reduction of the ventricular width due to shunts in patients with communicating hydrocephalus seems to be unphysiological and does not correlate with a better outcome. ${ }^{[18]}$

Finding one is much more interesting, since the development of the ventricular width after EVD was a predictor for successful VP-shunt therapy in the later course of disease. Possibly, this could allow anticipatory identification of patients who need specific valve types or valve settings and higher follow-up frequency.

The interpretation of this finding could be that $\mathrm{pHC}$ after aneurysm rupture is not a homogeneous entity. Thus, patients who would have developed chronic hydrocephalus at a later stage and independently from the aneurysm rupture may develop a type of $\mathrm{pHC}$, which is more similar to chronic hydrocephalus, for example, iNPH. Possibly, the pathophysiology of this subgroup is more depended on CSF pulsatility and does not result in major changes of the EI due to external CSF drainage. . $^{[7,19]}$ The shorter IESS of this subgroup could be explained by the fact that these patients would actually require more sophisticated preoperative diagnostics, for example, CSF infusion studies in order to define the right valve type and pressure setting. 
On the other hand, the pathophysiology of the subgroup of patients who showed a greater change of EI after EVD could be more similar to acute pressure hydrocephalus and thus, be more tolerant concerning the valve type and setting.

This interpretation is supported by the findings of Yoshioka et al. ${ }^{[10]}$ who reported the incidence of $\mathrm{pHC}$ after aneurysmatic SAH increasing significantly with age and found high incidences even after mild SAH in elderly patients. This underlines the hypothesis of a proportion of SAH patients who bear a hydrocephalic precondition which is onset by the SAH. Unfortunately, our patient collective was too small to substantiate a correlation between baseline parameters, the reaction to external CSF drainage and the IESS.

\section{Valve type versus initial equalized shunt survival}

The ideal valve to regulate CSF drainage after aneurysmal $\mathrm{SAH}$ would be draining CSF in a mid-pressure range between 70 and $120 \mathrm{~mm} \mathrm{H}_{2} \mathrm{O}$ and would offer a high tolerance with regard to protein content in CSF. Till date, the quest of an ideal valve among the available valves for shunt dependent $\mathrm{pHC}$ is still going on.

In this study, by trend, the patient group who received a flow-regulated valve showed longer IESS. Nevertheless, this was only a trend since Kaplan-Meyer survival curves analyzing IESS in the different valve groups did also not show any statistical difference. Shunt placement with differential pressure valves took place significantly after a shorter interval after SAH compared to the other groups $(P=0.001)$. Most probably this was the fact because the 9 patients who were admitted for shunting in a secondary setting were shunted using either flow (33\%) or gravitational assisted valves $(55 \%)$.

There are numerous studies analyzing the risk factors for shunt dependency in pHC. ${ }^{[2,3]}$ However the literature does not provide much useful data for neurosurgeons choosing a valve type when shunting a patient with a $\mathrm{pHC}$ and also third ventriculostomy in $\mathrm{pHC}$ did not show promising results. ${ }^{[20,21]}$

\section{Limitations}

It is difficult to draw conclusions from this study since the study cohort is retrospectively analyzed. A prospective analysis would reduce confounding effects resulting from the treatment course of the patients. Furthermore, some of the analyzed parameters have a significant haziness, for example measurement of EI has an inter-rater variability. The clinical course of patients who survive acute aneurysmatic SAH can be extremely variable. This becomes especially true while deciding which valve to implant in a patient who is dependent on permanent CSF drainage. For example, an adjustable differential pressure valve might be chosen in a bedridden patient who might initially profit from a low-pressure valve of $100 \mathrm{~mm} \mathrm{H}_{2} \mathrm{O}$. Being in rehabilitation, the patient is mobilized and might develop CSF over-drainage resulting in subdural hygroma. This might either reflect too low adjustment of the programmable valve or the need of a gravitational unit.

One might think that the follow-up period of 6 months might be too short to analyze IESS. However, since it has been shown that shunt related complications in $\mathrm{pHC}$ occur within the first 6 months $(80 \%)$. This cut off value makes sense. ${ }^{[12]}$

\section{Conclusion}

Decline of the ventricular width after EVD placement was a predictor for successful VP-shunt therapy in the later course of disease in patients with pHC after aneurysmal SAH. Possibly, this could allow anticipatorily identifying patients who benefit from simple nonprogrammable valves and thus can avoid complications associated with complex valve types.

Bearing this in mind, valve type in patients must be tested in a larger prospective randomized trial to identify which valve might be needed in which patient.

\section{Financial support and sponsorship}

Nil.

\section{Conflicts of interest}

There are no conflicts of interest.

\section{References}

1. de Oliveira JG, Beck J, Setzer M, Gerlach R, Vatter H, Seifert V, et al. Risk of shunt-dependent hydrocephalus after occlusion of ruptured intracranial aneurysms by surgical clipping or endovascular coiling: A single-institution series and meta-analysis. Neurosurgery 2007;61:924-33.

2. Dorai Z, Hynan LS, Kopitnik TA, Samson D. Factors related to hydrocephalus after aneurysmal subarachnoid hemorrhage. Neurosurgery 2003;52:763-9.

3. Black PM. Hydrocephalus and vasospasm after subarachnoid hemorrhage from ruptured intracranial aneurysms. Neurosurgery 1986;18:12-6.

4. Dehdashti AR, Rilliet B, Rufenacht DA, de Tribolet N. Shunt-dependent hydrocephalus after rupture of intracranial aneurysms: A prospective study of the influence of treatment modality. J Neurosurg 2004;101:402-7.

5. Hirashima Y, Kurimoto M, Hayashi N, Umemura K, Hori E, Origasa H, et al. Duration of cerebrospinal fluid drainage in patients with aneurysmal subarachnoid hemorrhage for prevention of symptomatic vasospasm and late hydrocephalus. Neurol Med Chir (Tokyo) 2005;45:177-82.

6. Tian HL, Xu T, Hu J, Cui YH, Chen H, Zhou LF. Risk factors related to hydrocephalus after traumatic subarachnoid hemorrhage. Surg Neurol 2008;69:241-6. 
7. Greitz D. Radiological assessment of hydrocephalus: New theories and implications for therapy. Neurosurg Rev 2004;27:145-65.

8. Kazumata K, Kamiyama H, Ishikawa T. Reference table predicting the outcome of subarachnoid hemorrhage in the elderly, stratified by age. J Stroke Cerebrovasc Dis 2006;15:14-7.

9. Kazumata K, Kamiyama H, Ishikawa T, Makino K, Nakamura T, Takizawa K, et al. Clinical study of cerebrospinal fluid dynamics using 111In-DTPA SPECT in patients with subarachnoid hemorrhage. Neurol Med Chir (Tokyo) 2006;46:11-7.

10. Yoshioka H, Inagawa T, Tokuda Y, Inokuchi F. Chronic hydrocephalus in elderly patients following subarachnoid hemorrhage. Surg Neurol 2000;53:119-24.

11. Prusseit J, Simon M, von der Brelie C, Heep A, Molitor E, Volz S, et al. Epidemiology, prevention and management of ventriculoperitoneal shunt infections in children. Pediatr Neurosurg 2009;45:325-36.

12. Reddy GK. Ventriculoperitoneal shunt surgery and the incidence of shunt revision in adult patients with hemorrhage-related hydrocephalus. Clin Neurol Neurosurg 2012;114:1211-6.

13. von der Brelie C, Simon A, Gröner A, Molitor E, Simon M. Evaluation of an institutional guideline for the treatment of cerebrospinal fluid shunt-associated infections. Acta Neurochir (Wien) 2012;154:1691-7.

14. Evans WA. An encephalographic ratio for estimating ventricular enlargement and cerebral atrophy. Arch Neurol Psychiatry 1942. p. 931-7.

15. Lemcke J, Meier U, Müller C, Fritsch MJ, Kehler U, Langer N, et al. Safety and efficacy of gravitational shunt valves in patients with idiopathic normal pressure hydrocephalus: A pragmatic, randomised, open label, multicentre trial (SVASONA). J Neurol Neurosurg Psychiatry 2013;84:850-7.

16. Meier U, Stengel D, Müller C, Fritsch MJ, Kehler U, Langer N, et al. Predictors of subsequent overdrainage and clinical outcomes after ventriculoperitoneal shunting for idiopathic normal pressure hydrocephalus. Neurosurgery 2013;73:1054-60.

17. Meier U, Mutze S. Correlation between decreased ventricular size and positive clinical outcome following shunt placement in patients with normal-pressure hydrocephalus. J Neurosurg 2004;100:1036-40.

18. Esposito DP, Goldenberg FD, Frank JI, Ardelt AA, Roitberg BZ Permanent cerebrospinal fluid diversion in subarachnoid hemorrhage: Influence of physician practice style. Surg Neurol Int 2011;2:117.

19. Qvarlander S, Lundkvist B, Koskinen LO, Malm J, Eklund A. Pulsatility in CSF dynamics: Pathophysiology of idiopathic normal pressure hydrocephalus. J Neurol Neurosurg Psychiatry 2013;84:735-41.

20. Fukuhara T, Shimizu T, Namba Y. Limited efficacy of endoscopic third ventriculostomy for hydrocephalus following aneurysmal subarachnoid hemorrhage. Neurol Med Chir (Tokyo) 2009;49:449-55.

21. Gonda DD, Kim TE, Warnke PC, Kasper EM, Carter BS, Chen CC. Ventriculoperitoneal shunting versus endoscopic third ventriculostomy in the treatment of patients with hydrocephalus related to metastasis. Surg Neurol Int 2012;3:97. 